\title{
Philosophiques
}

\section{Peuples et territoires}

\section{Michel Seymour}

Volume 39, numéro 2, automne 2012

Territorialité, identité nationale et justice mondiale

URI : https://id.erudit.org/iderudit/1013690ar

DOI : https://doi.org/10.7202/1013690ar

Aller au sommaire du numéro

\section{Éditeur(s)}

Société de philosophie du Québec

\section{ISSN}

0316-2923 (imprimé)

1492-1391 (numérique)

Découvrir la revue

\section{Citer cet article}

Seymour, M. (2012). Peuples et territoires. Philosophiques, 39(2), 353-365. https://doi.org/10.7202/1013690ar

\section{Résumé de l'article}

Dans ce texte, j'examine sur un mode programmatique la relation qui existe entre les peuples et les territoires. Les frontières des peuples souverains sont-elles sacrées, naturelles et absolues, voire irréfragables ? Le territoire a-t-il une importance identitaire ? Si oui, cette relation identitaire repose-t-elle sur l'attachement sentimental des citoyens ou sur une préférence rationnelle? Doit-on plutôt l'expliquer par un rapport historique ? Le territoire est-il un élément constitutif de l'identité d'un peuple ? Le principe de l'intégrité du territoire a-t-il une priorité absolue sur le principe affirmant le droit à l'autodétermination des peuples ? Tel est l'éventail de questions qui peuvent être posées en ce qui concerne la relation entre les peuples et leurs territoires. Je veux présenter une perspective qui me semble être originale. Dans la perspective du libéralisme politique, je pars d'une conception institutionnelle du peuple. Je me propose d'indiquer ensuite comment cette approche permet d'envisager des réponses à ces questions.
Ce document est protégé par la loi sur le droit d'auteur. L’utilisation des services d’Érudit (y compris la reproduction) est assujettie à sa politique d'utilisation que vous pouvez consulter en ligne.

https://apropos.erudit.org/fr/usagers/politique-dutilisation/ 


\title{
Peuples et territoires
}

\author{
MICHEL SEYMOUR \\ Département de philosophie \\ Université de Montréal
}

\begin{abstract}
RÉSUMÉ. - Dans ce texte, j'examine sur un mode programmatique la relation qui existe entre les peuples et les territoires. Les frontières des peuples souverains sont-elles sacrées, naturelles et absolues, voire irréfragables? Le territoire a-t-il une importance identitaire? Si oui, cette relation identitaire repose-t-elle sur l'attachement sentimental des citoyens ou sur une préférence rationnelle? Doit-on plutôt l'expliquer par un rapport historique? Le territoire est-il un élément constitutif de l'identité d'un peuple? Le principe de l'intégrité du territoire a-t-il une priorité absolue sur le principe affirmant le droit à l'autodétermination des peuples? Tel est l'éventail de questions qui peuvent être posées en ce qui concerne la relation entre les peuples et leurs territoires. Je veux présenter une perspective qui me semble être originale. Dans la perspective du libéralisme politique, je pars d'une conception institutionnelle du peuple. Je me propose d'indiquer ensuite comment cette approche permet d'envisager des réponses à ces questions.
\end{abstract}

\begin{abstract}
In this paper, I examine in a programmatic fashion the relationship between peoples and territories. Are the borders of sovereign peoples sacred, natural and absolute, or even irrebuttable? Does territory plays an important role for identity? If so, is this relationship based on the sentimental attachment of citizens or on a rational preference? Should it be explained instead by a historical relationship? Is territory even constitutive of the identity of a people? Does the principle of territorial integrity have priority over the principle asserting the right to self-determination of peoples? Such are the issues that can be raised concerning the relationship between peoples and their territories. I want to present an account that I take to be original. In accordance with political liberalism, I start from an institutional conception of peoples. I then indicate how this approach allows to consider answers to some of these questions.
\end{abstract}

Je me propose de soulever un certain nombre de questions concernant la relation qui existe entre les peuples et leurs territoires. Les frontières des peuples souverains sont-elles sacrées, naturelles et absolues, voire irréfragables? Le territoire a-t-il une importance identitaire? Si oui, cette relation identitaire repose-t-elle sur l'attachement sentimental des citoyens ou sur une préférence rationnelle? Doit-on plutôt l'expliquer par un rapport historique? Le territoire est-il constitutif de l'identité d'un peuple? Le territoire et les ressources naturelles sont-ils des objets de propriété privée, de propriété collective ou de propriété étatique? Doit-on plutôt dire, dans une perspective cosmopolitique, que les ressources naturelles appartiennent en fait à l'humanité entière? La souveraineté implique-t-elle un droit de propriété à l'égard d'un territoire spécifique, ou le territoire a-t-il seulement une valeur instrumentale pour l'exercice des pouvoirs associés à un État souve-

\section{PHILOSOPHIQUES 39/2 - Automne 2012, p. 353-365}


rain? Le principe de l'intégrité du territoire a-t-il une priorité absolue sur le principe affirmant le droit à l'autodétermination des peuples? Tel est l'éventail de questions qui peuvent être posées en ce qui concerne la relation entre les peuples et leurs territoires. Je veux présenter une perspective qui me semble être originale et indiquer ensuite comment celle-ci permet d'envisager des réponses à ces questions. Il s'agit en l'occurrence de prendre appui sur une conception institutionnelle du peuple, telle qu'elle s'impose dans le cadre du libéralisme politique. Il n'y a à mon avis aucun espoir de voir plus clair dans la relation entre les peuples et le territoire si l'on ne se prononce pas dès le départ très clairement au sujet de la notion de peuple.

\section{Définir cequ'est un peuple}

On pourrait tout d'abord faire valoir le fait qu'il existe plusieurs sortes de peuples, correspondant à divers types de conscience nationale. D'ailleurs, la pluralité de peuples explique pourquoi il est si difficile de produire une définition unique. J'ai ainsi montré ailleurs qu'il existait des peuples ethniques (fondés sur une origine ancestrale commune présumée, comme chez certains peuples autochtones); des peuples culturels (c'est-à-dire des sociétés multiethniques fondées sur le partage d'une langue, d'institutions et d'un patrimoine commun sans organisation gouvernementale, comme le peuple acadien); des peuples civiques (des sociétés organisées en États souverains mononationaux comme le Portugal, l'Islande ou les deux Corée); des peuples multisociétaux (des sociétés souveraines contenant en leur sein plusieurs peuples minoritaires, comme le Canada, la Grande-Bretagne et l'Espagne); des peuples sociopolitiques (des sociétés non souveraines mais formant des communautés politiques sous-étatiques pluriculturelles et pluriethniques, parfois même plurinationales, et partageant une langue publique commune, des institutions publiques communes dans lesquelles cette langue est principalement parlée, et une histoire publique commune, qui est celle de ces mêmes institutions, comme c'est le cas au Québec, en Écosse et en Catalogne); des peuples diasporiques (des sociétés qui partagent une culture tout en étant fragmentées en plusieurs minorités éparpillées sur des territoires discontinus, comme l'ancienne diaspora juive avant la création d'Israël); et des peuples multiterritoriaux (des sociétés vivant sur un territoire continu qui traverse plusieurs États, comme le Kurdistan).

J'ai aussi proposé de distinguer les peuples et les fragments minoritaires de peuples (souvent appelés minorités nationales dans le droit international), qu'il s'agisse de diasporas ${ }^{1}$ contiguës (c.-à-d. des extensions de nations voisines, comme les Russes dans les pays baltes) ou de diasporas non

1. J'emprunte cette expression à Radha Kumar (2004). Une extension de nation voisine est une sorte de "diaspora", si par cette expression on entend qu'un peuple principalement concentré sur un territoire donné est dispersé à l'extérieur de ce territoire. La diaspora contiguë est un fragment minoritaire d'un peuple qui se trouve «du mauvais côté de la frontière». 
contiguës (c.-à-d. des communautés issues de l'immigration). Il faut préciser aussi que mon approche s'appuie sur le libéralisme politique, inspiré de John Rawls (1993, I999), qu'il faut distinguer du libéralisme de Kant et de Mill.

(i) En ce sens, il s'agit de prendre ses distances à l'égard de l'individualisme moral. Mais il faut tout autant prendre ses distances à l'égard des approches essentialistes, collectivistes et primordialistes de la nation. Le libéralisme politique de Rawls s'appuie plutôt sur une conception strictement institutionnelle et politique des personnes (conçues comme citoyens) et des peuples (conçus comme "sociétés»). Autrement dit, on évite de s'engager sur le plan métaphysique, ce qui permet de contourner les objections souvent adressées aux ontologies sociales problématiques, et on évite de trancher entre les ontologies morales individualistes et les ontologies communautariennes.

(ii) Le libéralisme politique reconnaît les personnes et les peuples comme des agents moraux distincts, et comme deux sources autonomes de réclamations morales valides, conduisant à la mise en place, dans la théorie idéale, de deux positions originelles mettant en jeu respectivement les représentants de citoyens et les représentants de peuples.

(iii) Enfin, la valeur fondamentale du libéralisme politique est celle de la tolérance-respect, et pas celle de l'autonomie individuelle. Il s'agit donc d'une théorie fondée sur une certaine forme de reconnaissance réciproque.

On parvient dans le cadre de cette nouvelle approche à justifier l'octroi de droits collectifs aux peuples, car ceux-ci peuvent aussi être des agents moraux distincts tout aussi respectables dans l'espace politique. J'ai montré par la même occasion les apories et insuffisances d'une théorie libérale qui chercherait à obtenir des résultats équivalents en s'appuyant sur l'autonomie des individus. Or, la typologie des peuples et des fragments de peuples à laquelle je suis parvenu fait intervenir implicitement des considérations sur le territoire que le présent texte vise à mettre en lumière. Je présente ici quelques hypothèses de travail.

\section{Lecritère o'occupation effective}

Un peuple peut concevoir de différentes façons son rapport au territoire. Il peut, par exemple, le concevoir comme une terre sacrée ou promise, ou alors comme un territoire ancestral ou une patrie historique. Mais nous avons dit que, dans le cadre du libéralisme politique, les seules composantes subjectives pertinentes étaient l'autoreprésentation de la population et le vouloirvivre collectif. On peut conclure en premier lieu qu'il n'y a donc pas de place pour une identité territoriale fondée sur des critères d'identification ayant recours à des mythes, un territoire sacré, une terre promise ou une quelconque identité narrative collective. Ces divers types de représentations 
existent et font souvent partie de l'imaginaire social, mais ils ne sont pas nécessaires à l'existence d'un peuple.

Nous considérons les peuples seulement sous l'angle de leur identité institutionnelle de "culture sociétale " (c'est-à-dire comme des structures de cultures incarnées dans des caractères de cultures [Kymlicka I995]). Or, en tant que biens communément partagés, les institutions existent sur des territoires. La deuxième conclusion importante découle de ce qui vient d'être dit. Si les peuples sont appréhendés à partir de leur(s) langue(s) publique(s) communes, à partir d'institutions publiques communes (économiques, sociales, culturelles, éducatives, juridiques et parfois même gouvernementales) ainsi qu'à partir d'une histoire publique commune, et que ces institutions du peuple sont en vigueur sur un territoire, il s'ensuit que l'occupation du territoire est un élément constitutif de l'identité des peuples.

Selon le point de vue que je souhaite examiner, le territoire du peuple découle de l'occupation effective de ce même territoire. Mais en fait, l'expression "occupation effective» ne signifie pas nécessairement une occupation physique par la population, car l'identité institutionnelle peut varier selon le type de peuple, et donc selon le type de conscience nationale. Ainsi, l'occupation effective du territoire des peuples ethniques, culturels, multiterritoriaux et diasporiques équivaut à l'occupation physique de leur population respective, puisque leur identité institutionnelle n'implique pas la présence d'institutions gouvernementales inclusives. Le critère d'occupation effective proposé par Margaret Moore (200I, I92-I93) s'applique ici sans problème. Mais l'occupation effective du territoire des peuples civiques, multisociétaux et sociopolitiques correspond au territoire entrant sous la juridiction d'unités administratives gouvernementales, car ces dernières contribuent à déterminer l'identité institutionnelle de ces peuples et font partie de la représentation que ces populations se font d'elles-mêmes.

Ainsi, la typologie que je propose permet une application souple et diversifiée du critère d'occupation effective. Les peuples possèdent les territoires qu'ils occupent effectivement, mais il peut s'agir d'une occupation physique par la population ou d'une occupation inclusive au sein d'une unité administrative. Ces deux types d'occupation du territoire varient selon que la conscience nationale présente au sein de la population est de type "ethnique ", au sens traditionnel de l'expression (à ne pas confondre avec la notion de "peuple ethnique» mentionnée plus haut), ou de type inclusif (souvent décrit comme "civique", encore une fois au sens traditionnel, et non comme dans l'expression "peuple civique " utilisée plus haut). L'occupation effective des peuples ethniques, culturels, diasporiques et multiterritoriaux correspond à l'occupation physique, alors que l'occupation effective des peuples civiques, multisociétaux et sociopolitiques coïncide avec l'étendue des unités administratives. Par exemple, les Canadiens ne se représentent pas comme des membres de la nation canadienne-anglaise, mais bien comme des membres de la nation canadienne tout court, qui comprend l'en- 
semble des citoyens canadiens. Nous devons respecter cette auto-représentation nationale. Il en va de même pour le peuple québécois, dès lors qu'une masse critique de la population conçoit sa propre nation comme englobant l'ensemble des citoyens du Québec.

\section{Les ressources naturelles congures comme «natural endowments» des pauples}

J'établis une analogie entre les arguments de Rawls concernant les talents naturels (natural endowments) des individus, et les arguments que l'on pourrait formuler en ce qui concerne les ressources naturelles des peuples. Pour Rawls, l'éventail des talents constitue un bien (un atout) de la collectivité (Rawls I987, I32, 209; Rawls 2004, I IO-III). Ce n'est pas une vérité objective ou une prémisse indépendante permettant de dériver le principe de différence. Au contraire, l'accord sur le principe de différence revient à exprimer notre attachement à la distribution des talents (Rawls 2004, I I I).

Selon le point de vue envisagé, les ressources naturelles sont des objets de propriété collective, et non des objets de propriété privée. Elles sont à l'origine de l'identité des peuples, de la même manière que les talents sont un élément constitutif de l'identité des personnes. Les talents des individus n'appartiennent pas à la société, tout comme les ressources des peuples n'appartiennent pas à l'humanité entière. Mais la diversité et la complémentarité des talents appartiennent à la société, en tant que bien commun qui s'exprime dans le principe de différence. De la même manière, l'humanité entière possède la diversité et la complémentarité des ressources naturelles comme si c'était un bien commun, et cela s'exprimerait, pace Rawls, dans un principe de différence applicable à l'échelle internationale.

Les peuples ont un droit de propriété sur les ressources, mais il faut qu'ils respectent les minorités qui se trouvent sur leur territoire, les principes de justice distributive internationaux, ainsi que les principes de développement durable. En outre, lorsqu'un peuple souverain englobe une nation autochtone, il doit partager les territoires et les ressources naturelles avec elle, sans quoi son droit aux ressources revêt un caractère d'illégitimité.

\section{Un droit temitorial conditionnel}

Le territoire que les peuples occupent effectivement est "leur» territoire, mais ce n'est pas nécessairement un territoire qu'ils ont le droit de posséder. Ils le possèdent de facto, mais au départ, ils ne le possèdent pas de jure. Selon le point de vue que je voudrais défendre, chaque peuple a un droit "primaire » à un territoire, c'est-à-dire un droit qu'ils ont en partant sans que cela ne soit le résultat d'une réparation ou d'un remède à une injustice passée. Ceci dit, les peuples ne peuvent légitimement prétendre à un droit territorial que s'ils satisfont aussi à des principes normatifs. En l'occurrence, en plus de devoir respecter les autres peuples et les territoires occupés par eux, ils doivent aussi reconnaître les droits collectifs de leurs propres mino- 
rités. C'est de cette manière qu'ils se soumettront au principe normatif fondamental du libéralisme fondé sur le respect à l'égard de tous les agents au sein de la société. Il est souvent affirmé que l'on mesure la valeur d'un nationalisme au traitement qu'il fait subir à ses minorités internes. Or c'est justement ce dont il est question ici. Il faut donc distinguer (i) la propriété de facto du territoire qui, comme on vient de le voir, est un élément constitutif de l'identité des peuples; (ii) le droit territorial (dominion) (ou propriété de jure) qui est un droit primaire; et (iii) le droit à la souveraineté sur ce territoire (imperium), qui est le droit de former un État souverain gouvernant une population donnée. Ces trois concepts doivent aussi bien sûr être distingués de la propriété privée d'un terrain, ou droit foncier.

La possession du territoire par un peuple est purement une affaire de statut, et non de performance, pour emprunter la distinction proposée par Avery Kolers (2009). En ce sens, le peuple possède son territoire de facto. Reconnaître à une population le statut de peuple, c'est la reconnaître sur un territoire donné. Le droit territorial, par contre, est le droit primaire qu'a un peuple d'avoir un territoire. Il concerne l'autodétermination interne d'un peuple. Ce droit doit aller de pair avec un ensemble d'autres principes normatifs, mais vaut en tant que tel comme un droit inaliénable. C'est une affaire de performance, et non de statut. Le droit à la souveraineté, quant à lui, est la prérogative d'une population se trouvant sur un certain territoire. C'est un droit à l'autodétermination externe. Il doit prendre la forme d'une juste cause. Il n'existe pas de droit primaire d'avoir un État souverain. L'existence de ce droit est conditionnelle à la satisfaction de principes normatifs.

Je distinguerais donc, avec Kofman (2009), le droit territorial et le droit à la souveraineté, mais pas pour les mêmes raisons que lui. De mon point de vue, il s'agit de deux droits distincts parce que l'un s'applique à un territoire, et l'autre à une population, l'un relève de l'autodétermination interne, l'autre de l'autodétermination externe, l'un est un droit primaire et l'autre n'est pas primaire.

Mais du point de vue de Kofman, le territoire n'a qu'une fonction instrumentale pour l'exercice de la souveraineté, et l'État, même respectueux de ses minorités, n'a pas le droit d'exiger le respect de l'intégrité de ses frontières. Je crois plutôt qu'il n'est pas nécessaire de concevoir le territoire comme une simple valeur instrumentale pour exercer la souveraineté. L'État souverain qui respecte ses minorités a le droit de continuer d'exercer sa souveraineté et le droit de maintenir son intégrité territoriale. Inversement, l'État qui ne respecte pas ses minorités perd le droit de continuer d'exercer sa souveraineté sur ces populations, et il perd aussi le droit de maintenir son intégrité territoriale. Ainsi, même si le droit à la souveraineté et le droit de propriété sur un territoire sont deux notions distinctes, un peuple souverain qui respecterait le principe du droit à l'autodétermination interne de ses minorités aurait le droit à la souveraineté et le droit de préserver l'intégrité de son territoire. 
Ces considérations révèlent le lien étroit qui existe entre la réflexion sur le territoire et celle qui concerne la définition des différentes sortes de peuples. Une population ne peut justifier une conception inclusive du peuple - laquelle inclut donc une population entière se trouvant sur le territoire d'une unité administrative donnée -, que si elle reconnaît les minorités qui se trouvent en son sein. Sans une telle reconnaissance, la conception inclusive est illégitime, et les minorités sont justifiées de s'exclure de cette unité administrative. Le territoire occupé de facto par le peuple englobant n'est pas, en de telles circonstances, un territoire que ce peuple a le droit de posséder. Inversement, si un peuple majoritaire reconnaît les minorités en son sein et accepte les conséquences constitutionnelles et institutionnelles qui découlent de cette reconnaissance, c'est l'auto-exclusion de ces minorités qui est illégitime. Et les territoires que ces minorités réclament comme étant le leur ne sont pas des territoires qu'elles ont le droit de posséder exclusivement.

\section{Contre le nationalisme traditionnel et l'individualisme}

On notera aussi que si notre approche normative doit le moindrement s'ajuster à la diversité irréductible des sortes de peuples, il apparaît pour le moins problématique de défendre l'idée que les frontières du peuple doivent coïncider avec celles du pays. Si notre typologie est empiriquement fondée, il $\mathrm{y}$ a alors un très grand nombre de peuples, et il devient absurde de proposer que tous devraient avoir leur propre État. Cela nous permet de nous distancier du nationalisme traditionnel tel que défini par Ernest Gellner $($ I983, I). La coïncidence entre les frontières des peuples et celles d'États souverains vaut pour les peuples civiques et multisociétaux, mais pas pour les autres sortes de peuples. Et puisqu'il existe un très grand nombre de peuples entrant dans les autres catégories précédemment introduites, on admet donc sur le plan normatif qu'un territoire puisse contenir plusieurs peuples, comme c'est le cas pour le territoire des peuples multisociétaux, et aussi parfois le cas en ce qui concerne le territoire des peuples sociopolitiques. Enfin, les frontières de certains autres types de peuples ne coïncident pas avec les frontières existantes. Les peuples diasporiques et multiterritoriaux débordent en effet les frontières officiellement reconnues. En somme, il faut rejeter le nationalisme traditionnel qui n'admet que le modèle de la nation civique (un pays, une frontière, un peuple).

Mais il importe également de rejeter aussi le point de vue individualiste selon lequel ce sont les individus qui possèdent les territoires. Les individus qui émigrent ne peuvent obliger les États à les laisser occuper leurs territoires (Carens 1987, 2000). La détermination des frontières n'est pas non plus le fait de décisions prises par les individus (Abizadeh 2008, 20I0a, 20IOb). Les penseurs cosmopolitiques qui soutiennent ces thèses reconnaissent bien sûr qu'il faut des États et des frontières, mais ils estiment souvent qu'il faut ajuster nos décisions à cet égard aux préférences individuelles, tant en ce qui concerne leur admission sur le territoire qu'en ce qui concerne 
la détermination des frontières. Je suis pour ma part favorable à ce que des instances supranationales puissent dans certaines circonstances se prononcer sur l'immigration et les frontières, mais pas seulement pour faire valoir les droits internationaux des individus - car il y a aussi le droit international des peuples, entendus dans l'un ou l'autre des sept sens introduits plus tôt. Grâce au libéralisme politique et à la possibilité de reconnaître un ordre de droit applicable à certains peuples, nous sommes en mesure de justifier le recours à des règles existantes qui font intervenir ou supposent des droits pour les peuples, comme, par exemple, la règle de l'uti possidetis juris. Cette règle supranationale sert à déterminer les frontières des États nouvellement créés. Elle stipule que le pays nouvellement créé conserve les délimitations territoriales qui prévalaient à l'intérieur de l'État englobant. Il existe en outre des conventions internationales qu'il faut respecter, comme le droit à l'autodétermination des peuples, de même que les principes de l'intégrité territoriale des États. Nulle part nous ne trouvons dans le droit international actuel d'affirmation selon laquelle la détermination des frontières pourrait ou devrait être fonction des préférences rationnelles des individus.

L'identité des peuples n'est pas, elle non plus, fonction du seul consentement individuel. Les peuples ont certes une dimension "subjective», et pas seulement «objective", car ils dépendent en partie de l'auto-représentation et du vouloir-vivre de la population, mais il s'agit d'attitudes partagées par une masse critique au sein d'une population, et non d'attitudes requérant le consentement individuel de chacun. Le plébiscite quotidien de Renan (I 882) est un vouloir-vivre collectif, et la conscience nationale est une autoreprésentation collectivement partagée. L'affiliation nationale n'est pas non plus une affaire de préférence rationnelle individuelle, pas plus qu'elle n'est un attachement sentimental (patriotisme, loyauté) ou une identification fondée sur des mythes ou une quelconque identité narrative collective. Les deux seuls ingrédients «subjectifs» pertinents à l'identité nationale sont l'autoreprésentation et le vouloir-vivre des membres, et les autres ingrédients qui y sont souvent associés doivent être exclus de notre définition. Or, les deux composantes retenues sont collectives, et pas seulement un agrégat d'autoreprésentations et de volontés individuelles. Il s'agit plutôt d'un agrégat d'interprétations portant sur l'identité et la volonté du peuple dans lequel les membres se conçoivent alors comme faisant partie d'un tout. Ce tout est cimenté par une identité institutionnelle.

En outre, même si je rejette le principe des nationalités en vertu duquel les frontières des peuples doivent toujours coïncider avec celles d'États souverains, je crois qu'il est encore possible de s'en tenir à une approche collectiviste de la relation entre les peuples et leurs territoires. Il est possible de le faire (i) parce que les entités collectives sont appréhendées comme des agents institutionnels distincts. C'est aussi (ii) parce que les membres, au sein de ces institutions, se conçoivent comme des parties d'un tout et que leurs autoreprésentations collectives sont des agrégats d'interprétations individuelles 
portant sur l'identité et la volonté du peuple. C'est aussi enfin (iii) parce que les institutions qui forment l'identité du peuple s'appliquent sur un territoire. Bref, grâce à la caractérisation institutionnelle des peuples et à la typologie diversifiée, il semble possible de ménager une voie médiane entre le nationalisme traditionnel et l'individualisme.

\section{Ledroit à l'autodétermination}

Ce sont les peuples qui exercent le droit à l'autodétermination. Les peuples ont un droit primaire à l'autodétermination interne (c.-à-d. le droit de se développer économiquement, socialement et culturellement, et celui de déterminer son statut politique à l'intérieur de l'État), et ils n'ont pas à satisfaire des conditions morales additionnelles spécifiques pour jouir de ce droit. Mais ils n'ont pas pour autant automatiquement un droit à l'autodétermination externe (le droit de posséder un État souverain). Ils doivent avoir des justifications morales additionnelles pour avoir le droit de posséder un État. Le droit à l'autodétermination externe n'est pas un droit primaire. C'est un droit qui ne se justifie que sous la forme d'une juste cause.

Si l'on considère tout d'abord le droit à l'autodétermination interne, on doit dire que le libéralisme politique permet de dériver ce droit. Car, en vertu du libéralisme politique, les peuples sont considérés comme ayant une identité institutionnelle. Ensuite, le principe du respect égal qui est dû à tous les agents dans l'espace politique induit par conséquent le respect à l'égard des peuples. Ceux-ci ont donc le droit de développer leur identité institutionnelle de peuple. Autrement dit, les peuples ont le droit de développer leurs institutions économiques, culturelles et sociales, et le droit de déterminer leur statut politique à l'intérieur de l'État dans lequel ils se trouvent. Or ce droit est justement le droit à l'autodétermination interne. L'autodétermination interne des peuples s'applique, que ceux-ci détiennent déjà ou non un État.

Quant au droit à l'autodétermination externe, il existe aussi deux cas de figure. Il y a les peuples qui n'ont pas d'État, et il y a les peuples qui en ont déjà un. Là encore, les principes de respect mutuel et de reconnaissance réciproque du libéralisme politique s'appliquent. Si les peuples n'ont pas déjà un État souverain, leur autodétermination externe se justifie seulement si l'État dans lequel ils se trouvent ne respecte pas leur droit à l'autodétermination interne. Le peuple qui détient déjà un État, quant à lui, n'a le droit de posséder cet État que s'il respecte le droit à l'autodétermination interne des peuples minoritaires qu'il contient. S'il ne respecte pas ce droit, il ne peut invoquer le respect de son intégrité territoriale pour bloquer un processus d'autodétermination externe exercé par un peuple minoritaire. En ce qui concerne l'ensemble de ces points, la position que je défends rejoint pleinement l'état actuel du droit international. En effet, dans l'état actuel du droit, il n'existe pas de droit positif justifiant la sécession pure et simple. Il faut des justifications morales (colonisation, oppression ou violation de l'autodéter- 
mination interne). Bien sûr, on admet aussi la doctrine de l'effectivité. En vertu de celle-ci, si un peuple choisit démocratiquement la voie de la souveraineté, respecte ses minorités et se voit reconnaître par la communauté internationale, le droit international va constater la souveraineté du nouvel État sans avoir à statuer sur l'existence d'une injustice subie par ce peuple. Mais on peut penser que la doctrine de l'effectivité est une sorte de solution provisoire qui tient compte du fait qu'il n'existe pas encore un ensemble définitif de règles internationales concernant la sécession, en plus d'être le moyen de s'adapter à des facteurs contingents, imprévus et ponctuels caractéristiques de la réalité politique.

La théorie de l'autodétermination externe que je défends se rapproche de la théorie de la sécession comme réparation ("Remedial only theory of secession "), mais elle s'en distingue sur un point capital, puisqu'elle n'admet pas la primauté de l'intégrité territoriale des États sur l'autodétermination des peuples, primauté qui est acceptée par plusieurs des théoriciens tels que Lea Brilmayer (I99I, 2000), Allen Buchanan (2004), Paul Gilbert (I998), Donald Horowitz (2000, chapitre 6) et Wayne Norman (2006). Pour eux, le peuple doté d'un État n'a certes pas un droit primaire à l'autodétermination externe, et donc pas un droit primaire à la préservation de son intégrité territoriale, mais les exigences nécessaires pour justifier la sécession d'un peuple contenu en son sein sont tellement élevées qu'on a l'impression que l'intégrité territoriale prime sur le droit à l'autodétermination interne. Chez Buchanan, par exemple, les peuples n'ont pas de droits à l'autodétermination interne, et c'est pourquoi la violation de l'autodétermination interne ne figure pas dans sa liste des justes causes nécessaires à la sécession. En ce qui me concerne, je n'accorde pas de priorité à l'intégrité territoriale par rapport au droit à l'autodétermination. Le peuple qui détient déjà un État doit respecter le droit à l'autodétermination interne des peuples minoritaires pour mériter le respect de son intégrité territoriale.

Les différences avec la version adoptée par Buchanan sont nombreuses. Les peuples se distinguent des autres groupes culturels parce qu'ils sont les sources ultimes de la diversité culturelle. Ce sont des sujets de droits collectifs, et pas seulement des sujets de droits différenciés par le groupe. Je crois en outre, contrairement à Buchanan, que les peuples sont les seuls à pouvoir réclamer le droit à l'autodétermination interne. Ensuite, ce droit est primaire. Enfin, la violation de ce droit constitue une juste cause pour revendiquer la sécession, cause qui n'est pas prise en considération par Buchanan.

Je me distancie cependant tout autant des théories du droit de sécession qui supposent qu'un peuple a le droit de violer l'intégrité territoriale d'un État, même sans justification réparatrice, et même si l'État respecte son droit à l'autodétermination interne. Je rejette donc les approches nationalistes d'Avishai Margalit, de Joseph Raz (I990), et de Kai Nielsen (I998), tout comme je rejette la version particulière du droit primaire à la sécession, fondée exclusivement sur le principe démocratique, défendue par Harry 
Beran (1984) et Christopher Wellman (I995, 2005). Le droit à l'autodétermination et le droit à l'intégrité territoriale doivent être équilibrés et non hiérarchisés.

\section{Le principedel'Uti possidetis juris}

Il convient aussi d'insister sur la distinction entre la justice transitionnelle et la justice en période normale. La théorie que je voudrais développer suppose qu'en période normale le respect de l'intégrité territoriale et de la souveraineté étatique est conditionné par une politique de la reconnaissance de l'État à l'égard des minorités se trouvant sur son territoire. Si l'État ne respecte pas le droit à l'autodétermination interne des peuples sans États, il perd son droit territorial et son autorité sur l'ensemble de la population. Cela justifie une démarche de sécession unilatérale. Dans ce cas, le respect de l'autodétermination interne l'emporte sur l'intégrité territoriale.

Mais il en va tout autrement lorsque l'on entre dans une période de transition au moment où se met en place l'autorité d'un nouvel État souverain. Il faut ici faire valoir la primauté d'un principe de stabilité pour assurer une transition sans turbulences. Il faut en ce sens appliquer le principe de l'Uti possidetis juris, en vertu duquel il est affirmé que les frontières antérieures de l'État sécessionniste seront aussi les frontières de l'État souverain nouvellement formé (Frank et al, I992; Kumar, I997a, I997b). Ce principe a été appliqué par la Cour internationale de justice lors de la décolonisation en Afrique, ainsi que dans les cas de dissolution de l'URSS, de la Tchécoslovaquie et de la Yougoslavie. C'est un principe qui n'a de valeur que dans le contexte de la justice transitionnelle.

$\mathrm{Si}$, en période normale, le respect de l'autodétermination interne conditionne le principe du respect de l'intégrité territoriale, en période de transition, le respect de l'autodétermination interne est conditionné par le respect de l'intégrité territoriale de l'État nouvellement formé. Je me distancie donc de la position défendue par Daniel Kofman (2009), Avery Kolers (2009) et Margaret Moore (I999, 200I), qui veulent étendre le droit à l'autodétermination respectivement aux «groupes d'identité culturelle territoriale» (Territorial Cultural Identity Groups), aux "communautés ethnogéographiques»(Ethnogeographical Communities) ou aux "groupes nationaux" (national groups), et qui reconnaissent un droit de sécession à de tels groupes même pendant la période de transition, lorsqu'un peuple accède à sa souveraineté. Les trois expressions qu'ils utilisent pour faire référence aux groupes nationalitaires sont des néologismes censés inclure autant les peuples que les fragments minoritaires de peuples (on pense ici surtout à des diasporas contiguës). Ces catégories ne correspondent à aucune catégorie juridique internationale reconnue, elles leur servent à accorder à des fragments minoritaires de peuples un statut équivalent à celui de peuple, ce qui n'est pas du tout admis en droit international. Elles conduisent aussi ces auteurs à admettre un droit automatique de sécession (rejeté par le droit international) 
pour les peuples et, dans certaines conditions, pour les fragments de peuples - droit qui, en outre, peut être exercé au moment où un peuple majoritaire accède à sa souveraineté. Il s'agit de positions qui, dans certains cas, conduisent à l'irrédentisme et qui contreviennent de multiples façons au droit international actuel.

Les fragments minoritaires de peuples ont eux aussi des droits collectifs de protection de leur langue et de leur culture. Mais si un peuple qui englobe de telles minorités, s'engage dans un processus d'accession à la souveraineté et ne respecte pas leurs droits, la solution n'est pas de morceler le territoire du nouvel État en accordant un droit de sécession à des fragments minoritaires de peuples, mais bien plutôt de faire en sorte que la communauté internationale ne reconnaisse pas le nouvel État.

\section{Condusion}

Telle est donc, sous forme d'esquisse, ce à quoi peut ressembler une théorie globale de la relation qui existe entre les peuples et leur territoire. Il s'agit bien évidemment d'une description programmatique, mais elle a l'avantage de démontrer l'ampleur du chantier qui s'ouvre à nous. Elle a aussi l'avantage de montrer les liens qui existent entre tous les sujets abordés, et d'indiquer une piste théorique possible qui semble pouvoir être défendue de façon cohérente.

\section{Références}

Abizadeh Arash. "Closed Borders, Human Rights, and Democratic Legitimation », in David Hollenbach (ed.), Driven from Home: Human Rights and the New Realities of Forced Migration, Washington, DC: Georgetown University Press, 2010a.

—. "Citizenship, Immigration, and Boundaries", D.S.A. Bell (ed.), Ethics and World Politics, Oxford, Oxford University Press, 2010b.

—. "Democratic Theory and Border Coercion: No Right to Unilaterally Control Your Own Borders ", Political Theory 36, I, 2008, 37-65.

Beran, Harry. "A Liberal Theory of Secession ", Political Studies, 32, I984, 2I-3 I.

Brilmayer, Lea. "Secession and Self-Determination: One Decade Later», Yale Journal of International Law, vol. 25, 283 et seq., 2000.

—. "Secession and Self-Determination: A Territorial Interpretation ", Yale Journal of International Law, I6, I991, I77-202.

Buchanan, Allen. Justice, Legitimacy, and Self-Determination, Oxford, Oxford University Press, 2004.

Carens, Joseph. Culture, Citizenship, and Community, Oxford, Oxford University Press, 2000.

- - "Aliens and Citizens: the Case for Open Borders ", Review of Politics, 49, 3 , I $987,25 \mathrm{I}-273$.

Franck, Thomas M., Rosalyn Higgins, Alain Pellet, Malcolm N. Shaw, Christian Tomuschat. The Territorial Integrity of Quebec in the Event of the Attainment of Sovereignty, Report prepared for Quebec's ministère des Relations internationales, I992. 
Gellner, Ernest. Nations and Nationalism, New York, Cornell University Press, I983.

Gilbert, Paul. The Philosophy of Nationalism, Boulder, Westview Press, I998.

Horowitz, Donald. Ethnic Groups in Conflict, Berkeley and Los Angeles, University of California Press, 2000.

Kofman, Daniel. "Territorial Justice and Self-Determination », Territory and Justice Network, [http://eis.bris.ac.uk/ plcdib/territory/papers.htm]. Workshop in Vinodolski, Croatia, October 2009.

Kolers, Avery. Land, Conflict, and Justice. A Political Theory of Territory, Cambridge, Cambridge University Press, 2009.

Kumar, Radha. Divide and Fall? Bosnia in the Annals of Partition, New York, Verso, I997a.

—. "The Troubled History of Partition ", Foreign Affairs, February, I997b.

—. "Settling Partition Hostilities: Lessons Learned, Options Ahead", in Michel Seymour (ed.), The Fate of the Nation-State, Montréal-Kingston, McGillQueen's University Press, 2004, 247-270.

Kymlicka, Will. Multicultural Citizenship, Oxford, Oxford University Press, I995.

Margalit, Avishai \& Joseph Raz. "National Self-Determination ", Journal of Philosophy, 87, 9, I990, 439-46I.

Moore, Margaret. The Ethics of Nationalism, Oxford, Oxford University Press, 2001 .

_. "Nationalist Arguments, Ambivalent Conclusions ", The Monist, 82, 3, I999, 469-490.

Nielsen, Kai. "Liberal Nationalism and Secession", in Margaret Moore (ed.), National Self-Determination and Secession, Oxford, Oxford University Press, I998, I03-I33.

Norman, Wayne. Negotiating Nationalism. Nation-Building, Federalism and Secession in the Multination State, Oxford, Oxford University Press, 2006.

Rawls, John. La justice comme équité, Montréal, Boréal, 2004.

—. The Law of Peoples, Cambridge, Mass., Harvard University Press, I999.

—. Political Liberalism, New York, Columbia University Press, I993.

—. Théorie de la justice, Paris, Éditions du Seuil, 1987.

Renan, Ernest. Qu'est-ce qu'une nation? Paris, Calmann Lévy, I 882.

Wellman, Christopher H. A Theory of Secession: The Case for Political Self-Determination, Cambridge, Cambridge University Press, 2005.

-. "A Defence of Secession and Political Self-Determination", Philosophy and Public Affairs, 24, 2, I995, I42-7I. 